\title{
MAGNETIC FIELD SUPPRESSION IN COLLISION-LESS SHOCKS GENERATED DURING THE EXPANSION OF A DENSE PLASMA INTO A RAREFIED MEDIUM
}

\author{
G. Sarri ${ }^{1}$, M.E. Dieckmann ${ }^{1}$, I. Kourakis ${ }^{1}$ and M. Borghesi ${ }^{1}$
}

\begin{abstract}
A two-dimensional numerical study of the expansion of a dense plasma through a more rarefied one is reported. The electrostatic ion-acoustic shock, which is generated during the expansion, accelerates the electrons of the rarefied plasma inducing a superthermal population which reduces electron thermal anisotropy. The Weibel instability is therefore not triggered and no self-generated magnetic fields are observed, in contrast with published theoretical results dealing with plasma expansion into vacuum.
\end{abstract}

\section{Introduction}

The collision of plasma clouds with different properties (such as temperature, density, and composition) is a fairly common phenomenon in nature (Gaisser 1990). The high degree of nonlinearity of such collisions facilitates the onset of collisionless shock waves (CSWs) (ONeil \& Coroniti 1999; Eliasson \& Shukla 2006). Purely electrostatic, nonrelativistic CSWs might be present in astrophysical scenarios of great interest such as microquasar systems (Mirabel et al. 1992) or around ultramassive stars (such as the Herbig Ae/Be stars, Herbig 1960) whose atmosphere has been demonstrated to be completely unmagnetized (Vink et al. 2002). CSWs can nowadays be recreated also in laboratory experiments comprising the interaction of a high energy laser pulse with a solid target (Kuramitsu et al. 2011; Romagnani et al. 2008). From the theoretical point of view, plasma expansion has been studied, both analytically and numerically, mostly in the assumption of expansion into vacuum (Sack \& Schamel 1987; Mora 2005; Mora \& Grismayer 2009; Thaury et al. 2010), leading to magnetized plasma fronts. Here, we give the first numerical demonstration that the presence of a rarefied ambient plasma can forbid magnetic field generation by acting as a suppressor for the Weibel instability (Weibel 1959; Palodhi et al. 2009; Stockem et al. 2010) at the plasma front, leading to a purely electrostatic evolution of the plasma (Sarri et al. 2011a; Sarri et al. 2011b).

${ }^{1}$ School of Mathematics and Physics, The Queen's University of Belfast, Belfast BT7 1NN, UK 


\section{Simulation parameters}

We simulated the expansion of a dense plasma into a more rarefied one, via a two-dimensional particle-in-cell code (Fried \& Wong 1966). This work follows from an earlier study, restricted to one spatial dimension, whose discussion can be found in (Sarri et al. 2010). The simulation box dimension is $L_{x} \times L_{y}=$ $540 \lambda_{D} \times 60 \lambda_{D}$ with $\lambda_{D}$ the Debye length of the rarefied plasma. The box is divided along $x$ into two halves. The interval $-L_{x} / 2<x<0$ contains a plasma with density $n_{0}=10^{17} \mathrm{~cm}^{-3}$ whereas the interval $0<x<L_{x} / 2$ contains a plasma with density $n_{0} / 100$. Both plasmas have an electron (proton) temperature of $1 \mathrm{keV}(10 \mathrm{eV})$. Temperatures and densities have been chosen in order to be comparable with the experimental conditions reported in (Romagnani et al. 2008) and of relevance to the accretion disks in micro- quasar systems (measured blackbody radiation consistent with the presence of keV electrons, Fender \& Belloni 2004). The full simulation domain is resolved by a grid with $4000 \times 440$ cells and all boundary conditions are periodic. 360 (160) computational particles per simulation cell are used to represent the dense (rarefied) plasma species. The simulation ran for approximately half a nanosecond.

\section{Suppression of the Weibel instability}

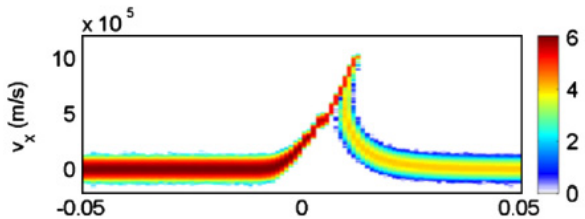

(a) $X(\mathrm{~mm})$

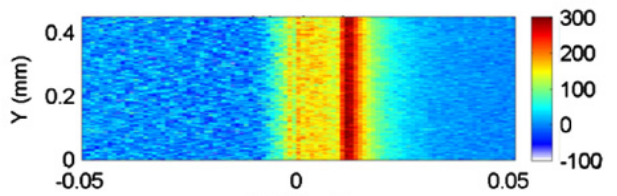

(c) $X(\mathrm{~mm})$

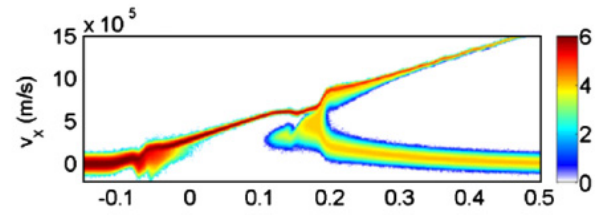

(b) $X(\mathrm{~mm})$

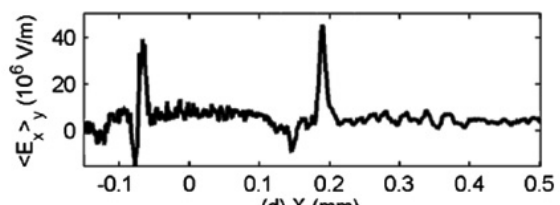

(d) $X(\mathrm{~mm})$

Fig. 1. Proton phase space distributions at $t_{1}=15 \mathrm{ps}$ (a) and $t_{2}=0.35 \mathrm{~ns}$ (b). The color scale is 10-logarithmic and in units of the average density of the tenuous plasma. (c) Spatial distribution of the electric field amplitude at $t_{1}=15$ ps. (d) Electric field distribution $E_{x}$, averaged over $y$ for $t_{2}=0.35 \mathrm{~ns}$. Electric fields are in unit of $10^{6} \mathrm{~V} / \mathrm{m}$.

The sharp density jump located at $x=0$ induces the electrons of the higher density plasma to immediately diffuse; the heavier protons are not able to keep up with this motion and a net charge imbalance is set close to the plasmas interface. The resulting positive electrostatic field (Fig. 1c) accelerates the protons of both plasmas leading to a bending in the proton phase space (Fig. 1a) which 
evolves into a shocklike structure that interrupts the rarefaction wave, approximately at $x=0.2 \mathrm{~mm}$ for $t_{2}=0.35 \mathrm{~ns}$ (Fig. 1b). This structure presents a monopolar electrostatic field (Fig. 1d) and propagates with a velocity of the order of the ion-acoustic speed of the tenuous plasma. An analogous structure is not observed in the electron phase space (compare Figs. 1b and 3a), corroborating the thesis of an ionic CSW. The most interesting result arising from direct analysis of
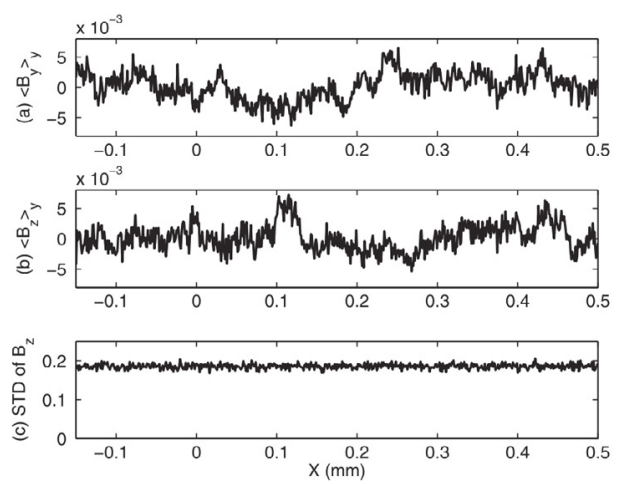

Fig. 2. Mean values of $B_{y}$ (a) and $B_{z}$ (b) Teslas, averaged along the $y$ direction, and the standard deviation of $B_{z}$ along the $y$ direction (c). All graphs refer to $t_{2}=0.35 \mathrm{~ns}$.

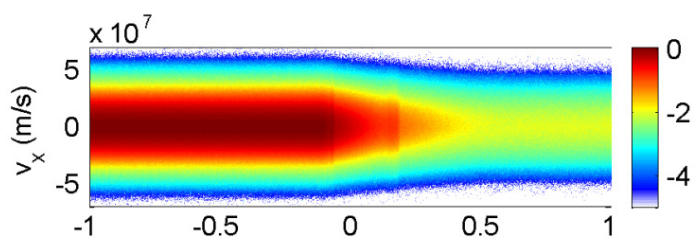

(a) $X(\mathrm{~mm})$

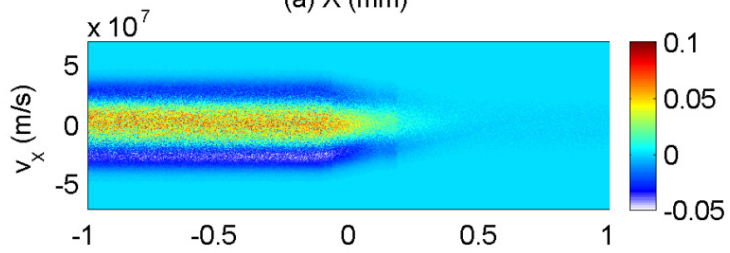

(b) $X(\mathrm{~mm})$

Fig. 3. (a) Electron phase space at $t_{2}=0.35$ ns. The color scale is 10 -logarithmic and in units of the average density of the dense plasma. (b) Difference between the electron distribution function at $t_{2}=0.35 \mathrm{~ns}$ and the initial Maxwellian distribution. The color scale is normalized to the peak of the distribution.

the simulation output is that no significant magnetic fields are generated throughout the entire simulation box (Fig. 2). The maximum fluctuation of the mean values of the magnetic $B_{y}$ and $B_{z}$ components are kept extremely low across the 
entire simulation box $\left(\left\langle B_{y}\right\rangle_{y},\left\langle B_{z}\right\rangle_{y} \leq 5 \times 10^{-3} \mathrm{~T}\right.$, see Figs. 2a and 2b) and always much smaller than the related standard deviation $(\approx 0.2 \mathrm{~T}$, see Fig. $2 \mathrm{c})$. The plasma dynamics, as well as the CSW generated, are therefore of purely electrostatic nature. This result is in contrast to simulations of plasma expansion into vacuum reported in literature (see, for instance, Thaury et al. 2010). During expansion into vacuum, the electrons of the plasma lose kinetic energy exclusively along the expansion axis, due to the transfer of energy from the electrons to the expanding protons. The electron velocity distribution develops thus an anisotropy which feeds the Weibel instability inducing a magnetized plasma front. However, if the plasma is forced to expand in a tenuous medium, a population of electrons is present ahead of the plasma front. Such a population is accelerated by the CSW and develops a superthermal distribution. These electrons reduce the gap between the thermal energy along the transverse and longitudinal directions and therefore limit the thermal anisotropy to be well below $10 \%$ for the entire duration of the simulation (see Fig. 3b). A thorough discussion of these numerical results can be found in (Sarri et al. 2011).

This work has been supported by the Engineering and Physical Sciences Research Council (Grant Numbers EP/E035728/1 (LIBRA consortium) and EP/D06337X/1) and by a Leverhulme Trust fellowship (ECF-2011-383).

\section{References}

Gaisser, T.K., 1990, Cosmic Rays and Particle Physics (Cambridge University Press, Cambridge, UK)

ONeil, T.M., \& Coroniti, F.V., 1999, Rev. Mod. Phys., 71, S404

Eliasson, B., \& Shukla, P.K., 2006, Phys. Rep., 422, 225

Mirabel, I.F., et al., 1992, Nature (London), 358, 215

Herbig, G.H., 1960, ApJS, 4, 337

Vink, J.S., et al., 2002, MNRAS, 337, 356

Kuramitsu, Y., et al., 2011, Phys. Rev. Lett., 106, 175002

Romagnani, L., et al., 2008, Phys. Rev. Lett., 101, 025004

Sack, C., \& Schamel, H., 1987, Phys. Rep., 156, 311

Mora, P., 2005, Phys. Rev. E, 72, 056401

Mora, P., \& Grismayer, T., 2009, Phys. Rev. Lett., 102, 145001

Thaury, C., et al., 2010, Phys. Rev. E, 82, 026408

Weibel, E.S., 1959, Phys. Rev. Lett., 2, 83

Palodhi, L., Califano, F., \& Pegoraro, F., 2009, Plasma Phys. Controlled Fusion, 51, 125006

Stockem, A., Dieckmann, M.E., \& Schlickeiser, R., 2010 Plasma Phys. Controlled Fusion, 52,085009

Sarri, G., et al., 2011a, Phys. Rev. Lett., 107, 025003

Sarri, G., et al., 2011b, New. J. Phys., 13, 073023

Fried, B.D., \& Wong, A.Y., 1966, Phys. Fluids, 9, 1084

Sarri, G., et al., 2010, Phys. Plasmas, 17, 082305

Fender, R., \& Belloni, T., 2004, A\&A, 42, 317 